4) In cortical sensory and motor lesions, the early components of SERs (wave $1,2,3)$ wer diminished in amplitude, showing their prolongation in latencies.

5) SERs would completely disappear in an extensive and advanced injuries of the cortex as well as in brain stem lesion with coma.

6) In the all cases of centrencephalic epilepsy, SERs showed conspicuously large negative wave with the peak around 170-200 msec. in latency, which were the characteristic late components bilaterally.

7) Wave 4 was inhibited in disturbances of motor area and would be well correlated with potentials of somatomotor cortex.

Clinical application of SERs would be great use combining with neurological and electroencephalographical findings.

\title{
F-8. Clinical Anplication of Somatosensory Evoked Potential Method in Neurosurgical Cases
}

\author{
Noboru Sakai, Keiichi Misawa and Kazuki Sakata \\ Second Department of Surgery, Gifu University School of Medicine
}

Clinical application of somatosensory evoked potnetial (SEP) examination in neurosurgical cases was studied. Bilateral ulnar nerves were simultaneously stimulated and bilateral SEPs were recorded bipolarly from the scalp surfaces correlsponding to somatosensory areas. Peak latency and ratio of amplitude between both sides were measured on various components of SEP in 33 normal subjects and in 45 cases of various organic brain diseases. Results obtained were as follows.

1) Forty-five cases of various orgaic brain diseases were classified into tumor $(\mathrm{T})$, hematoma $(\mathrm{H})$ and cerebrovascular lesion (CVL) groups. Peak latency tended to be prolonged in all 3 groups, as compared with normal control. This tendency was marked on the pathological side and, in T-group, it was especially marked in late components. Ratio of amplitude on the lesion side to that on the healthy side showed the following tendency. It was often larger than 1.0 in $\mathrm{N}_{1}$ and smaller than 1.0 in $\mathrm{N}_{2}$ in $\mathrm{T}$-group. In the other 2 groups, it was smaller than 1.0 in both $\mathrm{N}_{1}$ and $\mathrm{N}_{2}$, except that in some cases of chronic subdural hematoma the ratio of amplitude in $\mathrm{N}_{2}$ was almost equal to or larger than 1.0 .

2) T-group was divided into malignant and benign subgroups on the histological ground. Tendency of peak latency delay and tendencyof higher amplitude of $\mathrm{N}_{1}$ on the lesion side were more marked in the malignant subgroup.

3) From the point of view of tumor localization, SEP changes were most marked in the cases of parieto-temporal or thalamic tumors and no SEP change was observed in the cases of tumors in the chiasmal region.

4) In the cases of unilateral sensory disturbance of central origin, peak latencies of $N_{1}$ and $N_{2}$ tended to be prolonged and reduction in amplitudes of $N_{1}$ and $N_{2}$ (especially $\mathrm{N}_{1}$ ) on the lesion side was characteristic, except that in a few tumor cases 
$\mathrm{N}_{1}$ was of higher amplitude on the lesion side despite presence of sensory disturbance.

5) In the course of recovering sensory disturbance, it was generally observed that the amplitudes of $\mathrm{N}_{1}$ and $\mathrm{N}_{2}$ on the lesion side, which had been formerly reduced, increased to regian normal SEP pattern. This phenomenon was most typical in the cases of intracranial hematoma, especially of chronic subdural hematoma. In the tumor cases, in which $\mathrm{N}_{1}$ amplitude was higher on the lesion side despite presence of sensory disturbance, it was observed that $\mathrm{N}_{1}$ amplitude was quickly normalized following tumor extirpation in parallel with clinical improvement.

Studies on recovery function of human somatosensory evoked potential were performed in 10 normal control subjects and in 30 cases of posttraumatic chronic headache. Relationship between these results and scores of Cornell Medical Index (C. M. I. according to Fukamachi modification) was examined. Results obtained were as follows.

6) In control subjects, amplitude of SEP to test stimulus increased gradually as the interval between conditioning and test stimuli was increased from 50 to 300 msec. Approximate $100 \%$ revoery was attained at an inter-stimulus interval of 200 msec as for $\mathrm{N}_{1}$ and at $300 \mathrm{msec}$ as for $\mathrm{N}_{2}$ and $\mathrm{P}_{2}$.

7) Recovery function curve of $\mathrm{N}_{2}$ in posttraumatic headache cases, though $\mathrm{N}_{1}$ recovered grossly pallarel with control, showed any one of the following 3 types i) supernormal recovery at around $100 \mathrm{msec}$, ii) supernormal recovery at around 200 $\mathrm{msec}$ and iii) lower recovery function as a whole than the control. In most of the posttraumatic headache cases classified as area I and II of C. M. I., it was seen that facilitation was remarkablly present when the test stimulus was given 100 or 200 msec after the conditioning one. In most of the cases classified as area IV of C. M. I. no facilitation was present and values of recovery function gencrally were lower than the normal control. In the cases of area III of C. M. I., facilitation was or was not present. The above findings were observed also in $\mathbf{P}_{2}$.

Effects of stellate ganglion block on recovery function of SEP were examined. Recovery function at an inter-stimulus interval of $100 \mathrm{msec}$ in the cases of area $I$ and II of C. M. I. was compared with that in the cases of area IV of C. M. I., the latter serving as the control, and the following results were obtained.

8) In the control group, stellate ganglion block generally caused an increase in amplitude of $\mathrm{N}_{2}$. The cases of area I and II of C. M. I., in which stellate ganglion block was effective clinically, also showed similar tendency.

9) In the control group, in which the block was usually ineffective clinically, recovery function of $\mathrm{N}_{1}, \mathrm{~N}_{2}$ and $\mathrm{P}_{2}$ was not affected significantly by the block, while in the cases of area I and II in which the blockwas effective clinically, the phenomenon of supernormal recovery observed originally at an inter-stimulus interval of 100 msec disappeared and the recovery function was observed to be normalized after the block.

These results obtained were discussed. 